\title{
Multi-Band Linear Chirp Generation Based on a Type-III PLL
}

\author{
J. Velner*, E.A.M. Klumperink*, B. Nauta*, F.E. van Vliet* ${ }^{*}$ \\ ${ }^{*}$ University of Twente, 7500 AE, Enschede, The Netherlands ${ }^{\dagger}$ TNO, P.O. Box 96864, 2509 JG, The Hague, The Netherlands
}

\begin{abstract}
A type-III PLL is used to generate linear frequency ramps with $386 \mathrm{kHz}$ RMS frequency error in L-band $(3088 \mathrm{kHz}$ in X-band) and $10 \%$ fractional bandwidth. Pulse durations can be as low as $10 \mu \mathrm{s}$ without performance degradation. The system covers the L-, S-, C- and X-bands. A programmable output driver delivering -17.9 to $+4.9 \mathrm{dBm}$ differentially is included on chip. The system is fully integrated using a $250 \mathrm{~nm}$ SiGe BiCMOS process.
\end{abstract}

Index Terms-Phase locked loops, Ultra wideband radar, BiCMOS integrated circuits,

\section{INTRODUCTION}

Linear frequency ramps (chirps) are important for radar and imaging systems. A high bandwidth is necessary for a high range resolution [1]. For pulsed systems, a short chirp duration is needed to minimize the range below which no targets can be detected. This is because a short range corresponds to a short round trip time, hence a short pulse is required if the transmitter is to be deactivated when the echo is received. Flexibility in operating frequency is important, as lower frequency bands give a larger range and higher bands facilitate a larger bandwidth and thus a better resolution. Flexibility in output power can be helpful to taper an array aperture and to make sure the chip can be adapted to fit multiple systems with different drive power requirements. This paper presents a reconfigurable IC which can flexibly create very linear chirps in the commonly used L-, S-, C- and X-radarbands. Apart from FMCW signals, this system is also capable of transmitting a single frequency ramp after which the output is powered off. The IC achieves chirps down to $10 \mu \mathrm{s}$ in duration with $1 \mathrm{GHz}$ chirp bandwidth and $3088 \mathrm{kHz}$ RMS frequency error in X-band, or up to $125 \mathrm{MHz}$ bandwidth with $386 \mathrm{kHz}$ RMS error in L-band $\left(1 / 8^{\text {th }}\right.$ of the BW and error in $\mathrm{X}$-band). Through its single-chip nature, it opens the way to distributed radar transmitters.

This paper is divided into six sections. Section II describes the design on a system-level. Section III focuses on some circuit level aspects, with the first subsection discussing VCO design and the second subsection providing some details on part of the loop filter. Measurement results are discussed in section IV and a comparison with other work is made in section V. The final conclusions are treated in section VI.

\section{SySTEM OVERVIEW}

For flexibility reasons, we aim at frequency multiplication on a digitally created input chirp. Thus chirp speed and timing are digitally programmable. The challenge is to maintain a low RMS frequency error under such conditions. Frequency multiplication by $\mathrm{N}$ can be realized by an integer-N PLL. The

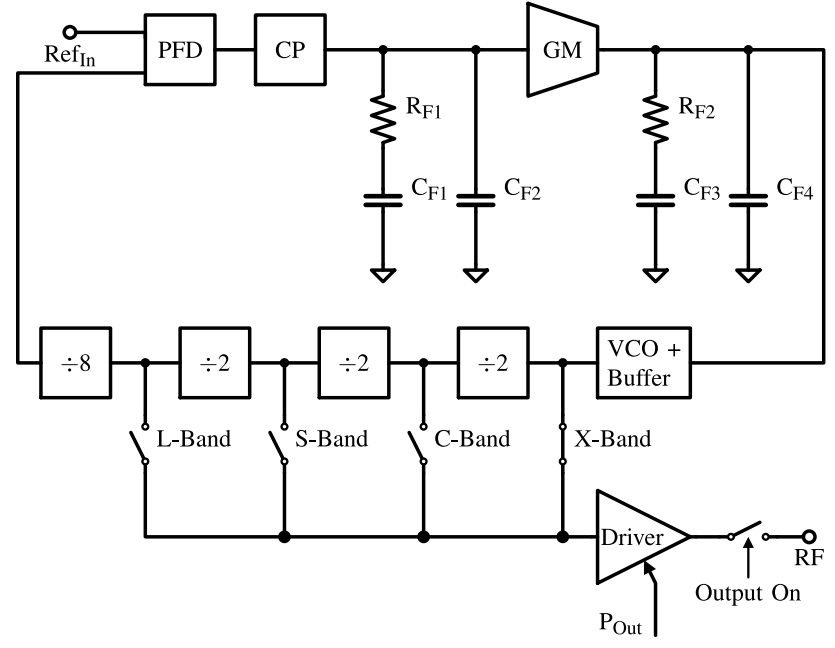

Fig. 1. IC Architecture

block diagram of the proposed system is shown in fig. 1 . The traditional structure of a PLL, consisting of a phase/frequency detector, filter, VCO and divider can be clearly distinguished. Additionally, there is a second stage in the loop filter (GM, $\mathrm{R}_{\mathrm{F} 2}, \mathrm{C}_{\mathrm{F} 3}$ and $\mathrm{C}_{\mathrm{F} 4}$ ). Through this filter stage, a type-III PLL loop is created. Although we did not find designs exploiting such a PLL in literature, it is known from control theory that systems with one ideal integrator (type-I) can track a constant input with zero error. Increasing the type increases the order of the input signal which can be perfectly tracked. Since a PLL has phase as its input signal, a type-III PLL can track a signal with quadratic phase vs. time (a linear frequency chirp), with zero error, regardless of its sweep rate.

A set of switches at the input of the output driver can be used to connect either the VCO buffer or one of the dividers to the output driver. This way the system can create chirps in the different frequency bands mentioned earlier. The output driver can be set at 16 different output voltage levels on a linear scale. The output is designed to deliver a maximum output power of $+6 \mathrm{dBm}$ and a minimum of $-18 \mathrm{dBm}$ at the lowest frequency bands and produce a square wave like signal. At higher frequencies both the fundamental and the harmonics will be filtered by the bondwires and board capacitances, yielding a sine wave like signal at lower power.

The pulsed chirps are created by applying a signal at $\operatorname{Ref}_{\text {in }}$ at a frequency slightly below the start frequency of the chirp and starting the chirp slightly before the output is activated, to avoid the settling behaviour of the loop. 


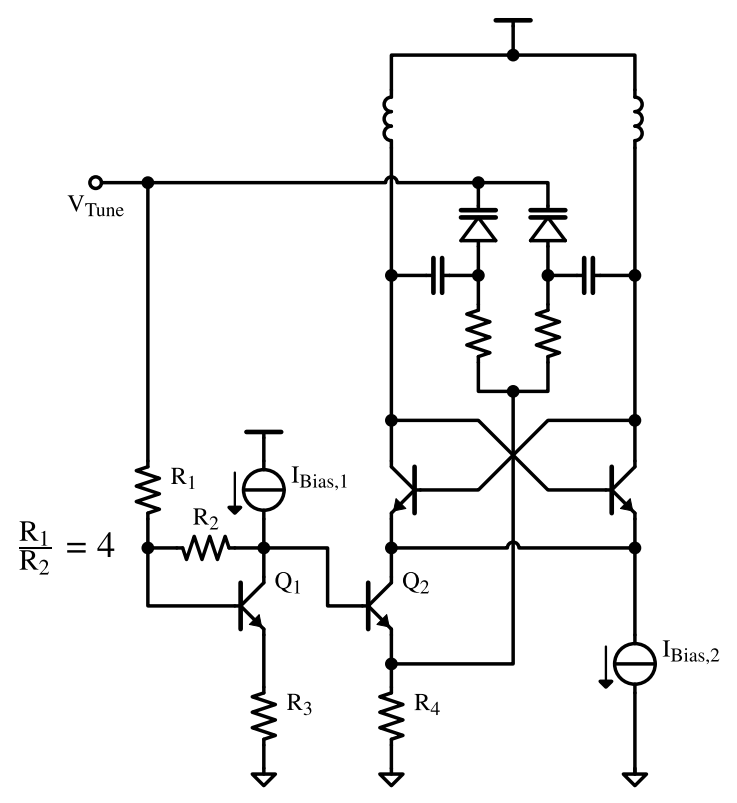

Fig. 2. Fast VCO Schematic

\section{Circuit Level Aspects}

\section{A. VCO Design}

An important circuit challenge in this system was to create a VCO that can be tuned over a wide enough range with available varicaps. In order to cover the entire X-band, two VCOs were used. One covers 7.2 to $10.1 \mathrm{GHz}$ and the other covers 8.8 to $12.2 \mathrm{GHz}$. A third VCO could extend the maximum frequency to over $16 \mathrm{GHz}$, allowing full coverage from 0.9 to $16 \mathrm{GHz}$. However, this VCO was not added in this proof-of-concept. The overlap between the VCOs is more than $1 \mathrm{GHz}$ to avoid VCO switching during a chirp.

Figure 2 shows the schematic of one of the VCOs. In this circuit, the tuning voltage is applied to an inverting amplifier, formed by $R_{1}-R_{3}$ and $Q_{1}$. This reduces the current trough $Q_{2}$ and $\mathrm{R}_{4}$ as the tuning voltage increases, maintaining a fairly constant output amplitude and avoiding saturation of the BJTs in the subsequent (CML) stage. The voltage across $R_{4}$ is also used to increase the voltage across the varicap. Simulations show that this increases the tuning range by about $20 \%$. The FoMs for both VCOs have been determined by simulation. Direct measurement is not possible because the phase noise and power of the VCOs cannot be measured directly. The traditional FoM for a VCO is calculated using (1) from [2].

$$
F o M=L(\Delta f)-20 \log \left(\frac{f_{0}}{\Delta f}\right)+10 \log \left(\frac{P}{1 m W}\right)
$$

A frequently used variation of this FoM takes the tuning range of the $\mathrm{VCO}$ into account by adding an additional factor [3]. This FoM $_{\mathrm{T}}$ can be found in (2).

$$
F_{0}=F o M-20 \log \left(\frac{T R}{10}\right)
$$

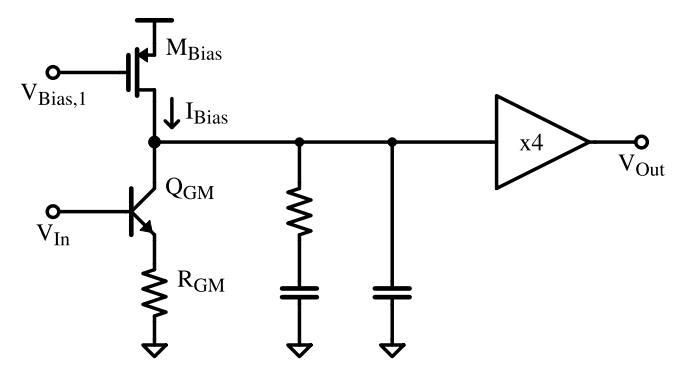

Fig. 3. Second Filter Stage Schematic

The simulations show a $\mathrm{FoM}_{\mathrm{T}}$ of $-181.8 \mathrm{dBc} / \mathrm{Hz}$ for both VCOs. This is comparable to, though not quite as good as, some designs that focus solely on VCO performance [3].

\section{B. Second Filter Stage}

A simplified schematic of the second filter stage, which turns the design into a type-III PLL, is shown in figure 3. As can be seen, its input consists of a transconductor created by $\mathrm{Q}_{\mathrm{GM}}$ and $\mathrm{R}_{\mathrm{GM}}$ which is biased by a constant current. This means that the circuit operates in class-A mode, where $\mathrm{Q}_{\mathrm{GM}}$ is always on (the circuit would clip if $\mathrm{Q}_{\mathrm{GM}}$ sinks more current than $\left.\mathrm{I}_{\mathrm{Bias}}\right)$. This was done to achieve a high bandwidth, operating the circuit in class- $\mathrm{AB}$ would require that the current through $\mathrm{M}_{\mathrm{Bias}}$ can be varied. Because the available MOSFETs are relatively slow, and the process has no PNP devices, this would seriously impact the bandwidth of the circuit, compromising the phase margin. The gain stage in figure 3 consists of an operational amplifier with a resistive feedback network giving it a gain of 4 . This was done to have an almost full scale voltage swing available at the VCO tuning node to maximise the tuning range. Without this gain stage the PMOS in the transconductor would go into triode for high tuning voltages and the bipolar device would go into saturation for low tuning voltages.

\section{MEASUREMENTS}

A chip micrograph of the IC, which was created in a $0.25 \mu \mathrm{m}$ BICMOS process with a bipolar $\mathrm{f}_{\mathrm{t}}$ of $140 \mathrm{GHz}$, can be found in fig. 4. The die measures $1.5 \mathrm{~mm}$ by $2 \mathrm{~mm}$.

The functionality of the chip has been verified by applying three reference signals and measuring the frequency versus time behaviour. All three reference signals result in a center frequency of $11.5 \mathrm{GHz}$ and a pulse duration of $10 \mu \mathrm{s}$, but the bandwidths differ. The first two signals are chirps with bandwidths of $1 \mathrm{GHz}$, and $100 \mathrm{MHz}$, the third has a constant frequency. Measurements have been performed by configuring the chip for L-band mode (see fig. 1) and measuring the output directly using an oscilloscope. This yields signals around $1437.5 \mathrm{MHz}$ with bandwidths of $125,12.5$ and $0 \mathrm{MHz}$ respectively. The signals were averaged 16 times to reduce quantization noise and band pass filtered using a filter with $60 \mathrm{~dB}$ out of band suppression and a passband between 1 and $2 \mathrm{GHz}$. The results, which can be found in fig. 5 show that the frequency error does not visibly change with chirp rate. 
TABLE I

COMPARISON TABLE

\begin{tabular}{|c|c|c|c|c|c|c|c|}
\hline Type & $\begin{array}{c}{[4]} \\
\text { FMCW }\end{array}$ & $\begin{array}{c}{[5]} \\
\text { FMCW }\end{array}$ & $\begin{array}{c}{[6]} \\
\text { FMCW }\end{array}$ & $\begin{array}{c}{[7]} \\
\text { FMCW }\end{array}$ & $\begin{array}{c}{[8]} \\
\text { FMCW }\end{array}$ & $\begin{array}{l}\text { This Work } \\
\text { Pulsed }\end{array}$ & \\
\hline Technology & $65 \mathrm{~nm}$ CMOS & $90 \mathrm{~nm}$ CMOS & $65 \mathrm{~nm}$ CMOS & SiGe Bipolar ${ }^{a}$ & Board & $250 \mathrm{~nm}$ BiCMOS & \\
\hline Active Area & 1.05 & 6.8 & 1.7 & $3^{a}$ & $?$ & 3 & $\mathrm{~mm}^{2}$ \\
\hline Supply Voltage & 1.2 & 1.2 & 1.2 & $5^{a}$ & $?$ & 2.5 & $\mathrm{~V}$ \\
\hline Power Consumption & $188^{b}$ & $406^{b}$ & 152 & $488^{a}$ & $?$ & $380^{c}$ & $\mathrm{~mW}$ \\
\hline Output Frequency & 75.6 to 76.3 & 78.1 to 78.8 & 82.1 to 83.8 & 67.2 to 92.8 & 76 to 78 & 7.2 to 12.2 divided by $1,2,4$ or 8 & $\mathrm{GHz}$ \\
\hline Chirp BW & 0.7 & 0.7 & 1.5 & 25.6 & 2 & $1^{d}$ & $\mathrm{GHz}$ \\
\hline Fractional Bandwidth & 1.3 & 1.3 & 2.3 & 32 & 2.6 & 10 & $\%$ \\
\hline RMS Frequency Error & $<300^{e}$ & $1050^{e}$ & 180 & $?$ & $?$ & $3088^{d}$ & $\mathrm{kHz}$ \\
\hline Chirp Time & $0.5^{f, g}$ & $0.25^{f, g}$ & 0.5 to $5^{f}$ & $4^{f}$ & $1^{f, g}$ & $\geq 0.01$ & $\mathrm{~ms}$ \\
\hline Output Power & 5.1 & -2.8 & $?$ & -3 to -1 & 10 & $-1 \overline{7} .9$ to 4.9 & $\mathrm{dBm}$ \\
\hline Phase Noise@1 MHz & -85.3 & -85 & -84 & -90 & $?$ & -86.0 to $-81.4^{d}$ & $\mathrm{dBc} / \mathrm{Hz}$ \\
\hline
\end{tabular}

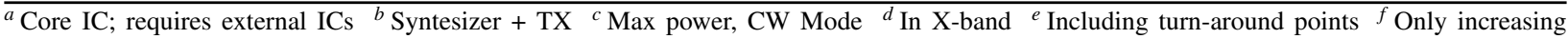
freq. part ${ }^{g}$ Lowest value reported

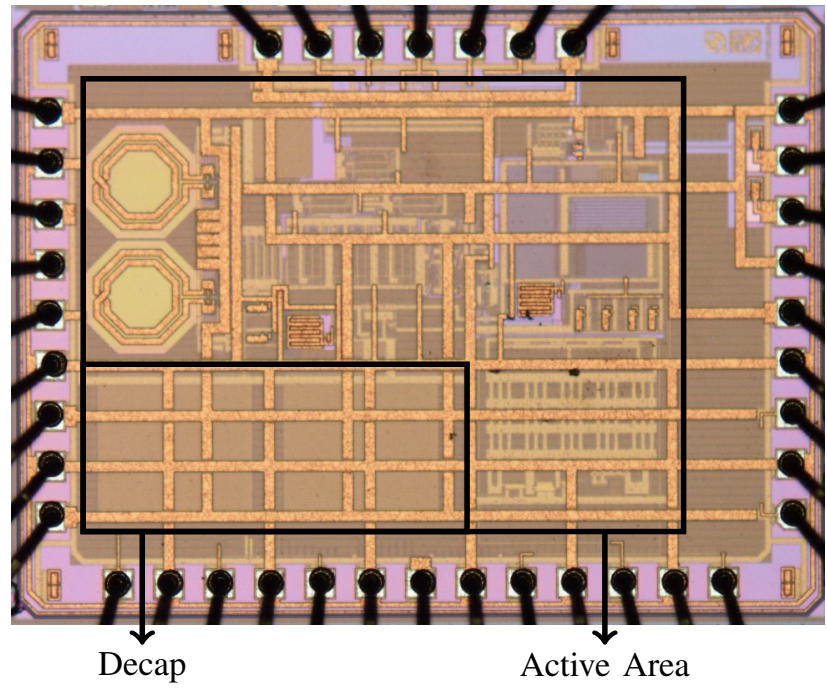

Fig. 4. Chip Micrograph

Figure 6 also shows the frequency error of the widest chirp (125 MHz) with respect to an ideal chirp. The RMS frequency error of both chirps is $386 \mathrm{kHz}$ and that of the constant frequency is $391 \mathrm{kHz}$, which confirms the independence of frequency error and chirp rate.

The frequency error in higher bands cannot be measured directly using available equipment, but since lower bands are obtained by dividing the output, the error in X-band is expected to be eight times higher than that at L-band, which is $3088 \mathrm{kHz}$. The measurements show a short transient, which is caused by powering up the output driver, at the start of the chirp. This transient was ignored for the frequency error calculation.

As the system corrects for any frequency error at every reference edge, the frequency error will result in reference spurs. Therefore, spur reduction will improve the performance for every chirp rate. The spurs in this design are at $-50 \mathrm{dBc}$ to $-58 \mathrm{dBc}$ in L-band and $-40 \mathrm{dBc}$ to $-45 \mathrm{dBc}$ in X-band. An improvement is expected with a redesign of the charge pump.

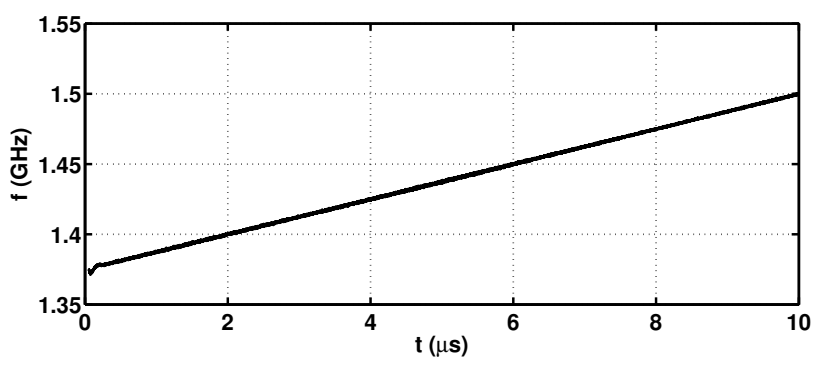

(a) $125 \mathrm{MHz}$ in $10 \mu \mathrm{s}$

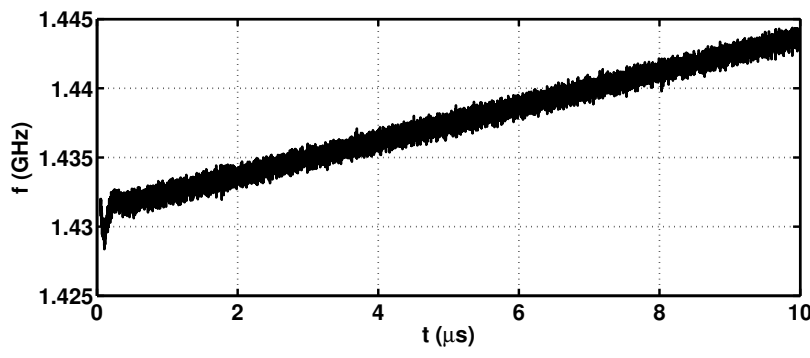

(b) $12.5 \mathrm{MHz}$ in $10 \mu \mathrm{s}$

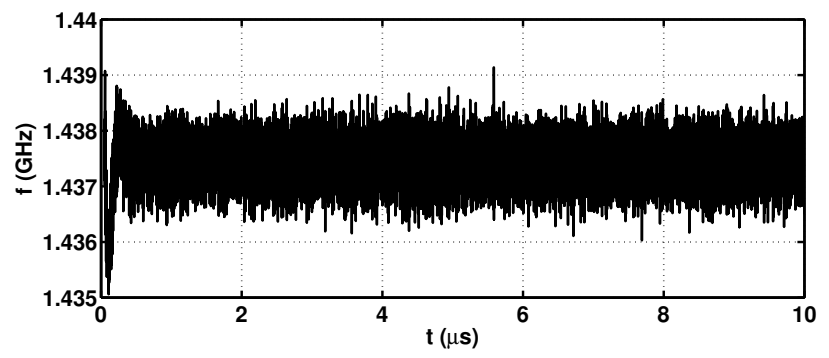

(c) Constant Frequency

Fig. 5. Frequency vs. Time 


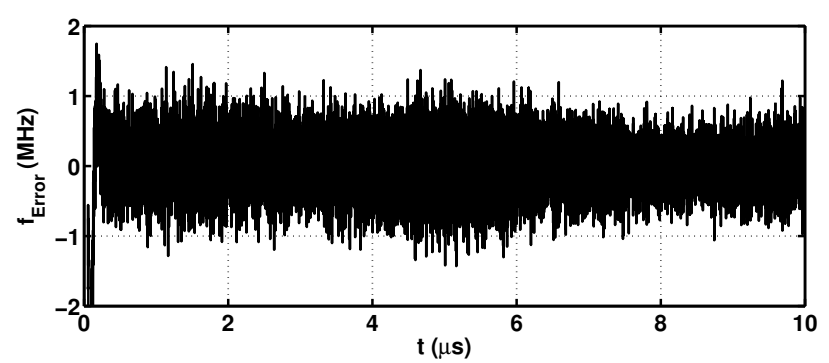

Fig. 6. Frequency Error

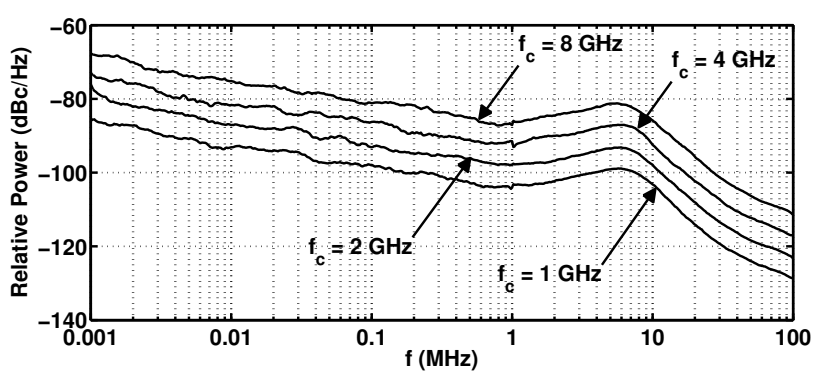

(a)

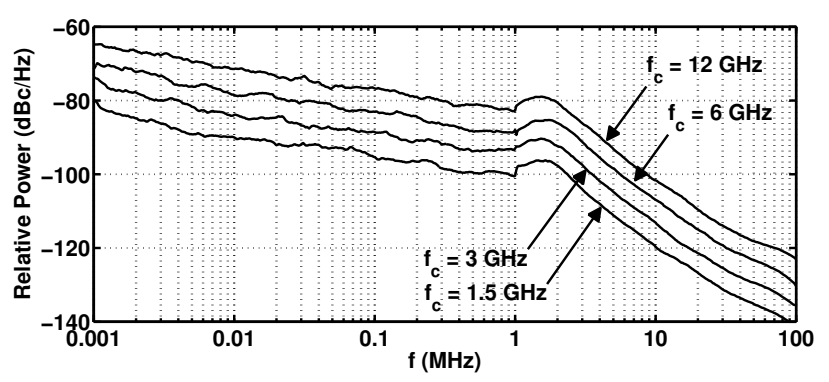

(b)

Fig. 7. Phase Noise

Figure 7 shows the phase noise of the system at each output band. Because the bandwidth of the system is so large, two plots are depicted here. Figure $7 \mathrm{a}$ depicts the phase noise of the slow VCO with the output frequency set at $8 \mathrm{GHz}$, fig. $7 \mathrm{~b}$ shows the phase noise of the fast VCO at $12 \mathrm{GHz}$. The difference between these plots can be explained by the fact that the fast VCO is more noisy than the slow VCO, but also by the difference in VCO gain for the given frequencies. This gain influences the loop dynamics, which explains the difference in cut-off frequency which is clearly visible.

The relatively high phase noise at $1 \mathrm{MHz}$ is also expected to be improved with a charge pump redesign. Due to the low $1 / \mathrm{f}$ corner of the bipolar devices, the phase noise is relatively low at small offset frequencies, which reduces range and Doppler inaccuracies. Furthermore, it allows for longer integration times and is hence beneficial for detecting slowmoving objects.

The output power was measured at -17.9 to $+4.9 \mathrm{dBm}$ in the lowest bands, dropping to -22.6 to $-0.7 \mathrm{dBm}$ at $12 \mathrm{GHz}$.
The output power is slightly lower than predicted because in practice it will not be an ideal square wave.

\section{COMParison to Other Work}

As the authors are not aware of previous publications of pulsed systems with this level of integration on silicon, a comparison is made with several FMCW systems. The real benefits, however, are present for fast-chirping pulse-Doppler systems. This comparison can be found in table I. As can be seen, the frequency error can be improved upon, but the chirp rate is by far the highest reported. Fractional bandwidth is surpassed only by [7], which has a far lower level of integration than this work.

\section{Conclusions}

A flexible system for the generation of pulsed linear frequency chirps has been presented. The system is capable of operation in L-, S-, C- and X-bands and achieves an RMS frequency error of $3088 \mathrm{kHz}$ for a $1 \mathrm{GHz}$ chirp in $10 \mu \mathrm{s}$ at $\mathrm{X}$-band. Due to the frequency multiplication in the system, the reference signal can be generated at low center frequency and bandwidth, allowing for digital generation.

\section{ACKNOWLEDGEMENTS}

The authors would like to thank NXP Semiconductors for silicon donation. Further, thanks are extended to Maurice van Wanum and Lex de Boer at TNO for their insights concerning the design and layout of the circuit. Finally the authors would like to thank Henk de Vries, Gerard Wienk, Mark Oude Alink, Bram Verhoef and Erik Olieman for invaluable assistance during the functional testing and characterization phases of the circuit.

\section{REFERENCES}

[1] M. Skolnik, Introduction to Radar Systems, McGraw-Hill, 2008.

[2] P. Baltus, et al., "A 3.5-mW, 2.5-GHz diversity receiver and a $1.2-\mathrm{mW}$, 3.6-GHz VCO in silicon on anything," IEEE J. Solid-State Circuits, vol. 33, no. 12, pp. 2074-2079, December 1998.

[3] Q. Wu1, et al., "A 10mW 37.8GHz Current-Redistribution BiCMOS VCO with an Average FOMT of $-193.5 \mathrm{dBc} / \mathrm{Hz}$," ISSCC Dig. Tech. Papers, pp. 150-151, February 2013.

[4] Y. Li, et al., "A Fully Integrated 77GHz FMCW Radar System in $65 \mathrm{~nm}$ CMOS," ISSCC Dig. Tech. Papers, pp. 216-217, February 2010.

[5] T. Mitomo, et al., "A $77 \mathrm{GHz} 90 \mathrm{~nm}$ CMOS Transceiver for FMCW Radar Applications," IEEE J. Solid-State Circuits, vol. 45, no. 4, pp. 928-937, April 2010

[6] H. Sakurai, et al., "A 1.5GHz-Modulation-Range 10ms-ModulationPeriod $180 \mathrm{kHz}$ RMS-Frequency-Error 26MHz-Reference Mixed-Mode FMCW Synthesizer for mm-Wave Radar Application," ISSCC Dig. Tech. Papers, pp. 292-294, February 2011.

[7] N. Pohl, et al., "An Ultra-Wideband $80 \mathrm{GHz}$ FMCW Radar System Using a SiGe Bipolar Transceiver Chip Stabilized by a Fractional-N PLL Synthesizer," IEEE Trans. Microw. Theory Tech., vol. 60, no. 3, pp. 757765, March 2012.

[8] C. Pfeffer, et al., "An IQ Modulator Based Heterodyne 77-GHz FMCW Colocated MIMO Radar System," MTT Digest, pp. 1-3, June 2012. 